\title{
Les multiples déterminants de la mémoire à court terme verbale : Implications théoriques et évaluatives.
}

\author{
Steve Majerus \\ Université de Liège (Belgique) \\ $\&$
}

Fonds de la Recherche Scientifique F.R.S.-FNRS (Belgique)

Version postscript auteur de:

Majerus, S. (2010). Les multiples déterminants de la mémoire à court terme verbale : implications théoriques et évaluatives. Développements, 4, 5-15.

\section{Adresse pour correspondance}

Steve Majerus

Centre de Recherches en Neurosciences Cognitives et Comportementales

Boulevard du Rectorat, B33, 4000 Liège

Tel : 003243664656

Fax : 003243662808

Email : smajerus@ulg.ac.be 


\section{ABSTRACT - FRANÇAIS}

Les tâches d'empan (empan de chiffres ; empan de mots) sont traditionnellement considérées comme mesurant les capacités limitées d'un système de mémoire à court terme verbale spécialisée tel que la boucle phonologique décrite par Baddeley et Hitch (1974). Cependant, de plus en plus de données montrent que ces tâches, en apparence très simples, recrutent en réalité des processus cognitifs complexes, en partie partagés avec d'autres fonctions cognitives. Parmi ces processus nous identifierons : (1) l'activation temporaire du système langagier, (2) l'attention sélective, (3) le traitement de l'ordre sériel. Nous proposerons un modèle théorique intégrant ces trois éléments comme principes de base de la mémoire à court terme verbale et de son développement chez l'enfant. Ensuite, nous développerons les implications pour l'évaluation de la mémoire à court terme verbale et les adaptations nécessaires afin de pouvoir mesurer l'intervention de chacun de ces trois éléments.

Mots clés : mémoire à court terme verbale, attention, ordre sériel, système langagier, acquisition du vocabulaire 


\section{ABSTRACT - ENGLISH}

Span tasks (digit span, word span) are considered to measure the limited capacity of a specialized verbal short-term memory system such as Baddeley and Hitch's (1974) phonological loop model. However, a vast amount of data suggests that these apparently simple tasks recruit complex cognitive processes, in part shared with other cognitive functions. We shall identity here three of these processes: (1) temporary activation of the language system, (2) selective attention, (3) serial order processing. We propose a theoretical model integrating these three processes as fundamental principles of verbal short-term memory and its development. We discuss the implications for the assessment of verbal shortterm memory and the adaptations that are necessary to distinguish the intervention of these three processes in verbal short-term memory tasks.

Mots clés : mémoire à court terme verbale, attention, ordre sériel, système langagier, acquisition du vocabulaire 


\section{POINT DE DÉPART : LA BOUCLE PHONOLOGIQUE}

Les tâches d'empan de chiffres ou d'empan de mots consistent dans des tâches de répétition verbale de séquences de chiffres ou de mots de longueur croissante. Le niveau d'empan reflète la longueur de la séquence la plus longue qui conduit à une répétition correcte pour $50 \%$ ou $75 \%$ des séquences de la même longueur présentées. Ainsi, l'empan de chiffres passe de 3 à l'âge de 4 ans à 6 à l'âge de 15 ans et atteint son maximum vers l'âge de 20 ans ; l'empan de mots est en général inférieur à l'empan de chiffres et passe de 2 à l'âge de 4 ans à 4 à l'âge de 15 ans (Gathercole, 2002). Traditionnellement, cette augmentation du niveau d'empan a été interprétée comme reflétant l'accroissement des capacités d'un système spécifique de la mémoire à court terme verbale, la boucle phonologique (Baddeley, 1986). La boucle phonologique s'articule autour de deux composants principaux : (1) le stock phonologique, de capacité limitée et permettant de stocker temporairement des informations verbales ; (2) la récapitulation articulatoire subvocale, permettant de réintroduire et de rafraichir les informations dans le stock phonologique, afin de contrecarrer la dégradation naturelle des informations stockées dans le stock phonologique. Au cours du développement, ces deux composants sont considérés augmenter de capacité et/ou d'efficacité, menant vers des performances de plus en plus élevées aux tâches d'empan. Par exemple, l'utilisation plus systématique de la récapitulation subvocale à partir de l'âge de 7 ans, et l'augmentation de la vitesse de récapitulation subvocale par la suite, expliquent une part non négligeable de l'augmentation du niveau d'empan de 7 à 15 ans (Cowan et al., 1998 ; Gathercole, 2002 ; Gathercole, Adams, \& Hitch, 1994 ; Hitch \& Halliday, 1983 ; Palmer, 2000). Cependant, de plus en plus de données montrent que les performances aux tâches d'empan ne dépendent pas uniquement de l'intervention d'un système hypothétique de mémoire à court terme verbale, mais peuvent s'expliquer par des facteurs plus généraux tels que l'intervention du système 
langagier et les capacités attentionnelles. L'objectif de cette revue de question sera de développer les données suggérant l'influence de ces facteurs plus généraux, de déterminer, face à ces données, dans quelle mesure le postulat d'un système spécifique de mémoire à court terme verbale reste encore nécessaire, et de proposer des amendements aux tâches de mémoire à court terme en fonction des nouveaux développements théoriques que nous aurons abordés.

\section{CONNAISSANCES LANGAGIÈRES ET MÉMOIRE A COURT TERME VERBALE}

Alors que la boucle phonologique a été longtemps considérée comme étant un système indépendant du système langagier, de nombreuses données montrent qu'en réalité, les connaissances langagières constituent un facteur déterminant de la mémoire à court terme. Ainsi, les connaissances langagières, que ce soit au niveau phonologique, lexical ou sémantique, vont déterminer la quantité de mots correctement rappelés dans les tâches de mémoire à court terme verbale. Parmi ces effets langagiers, l'effet de lexicalité est le plus important et se traduit par un meilleur rappel de listes de mots que de non-mots : alors que l'empan de mots avoisine les 5 mots chez l'adolescent, il peut ne pas dépasser les trois nonmots si les non-mots sont peu ressemblants à des mots existants (par exemple, /zøf, /pãb/, /tyr/) (Majerus \& Van der Linden, 2003). Cet effet a été interprété comme reflétant l'existence de connaissances lexicales s'activant lors de la présentation des mots à mémoriser et stabilisant les traces temporaires en mémoire à court terme par la suite, soit via un feedback permanent entre le système langagier et les traces en mémoire à court terme tout au long de la tâche, soit via une reconstruction des traces partiellement dégradées au moment du rappel (Hulme, Maughan, \& Brown, 1991 ; N. Martin \& Saffran, 1992 ; R. Martin, Shelton, \& Yaffee, 1994 ; Schweickert, 1993). Au niveau sous-lexical, des effets subtils impliquant la 
fréquence de co-occurrence des sons du langage ont également été démontrés : des non-mots composés de phonèmes fréquemment associés (i.e., /sॄftãs/) sont mieux rappelés dans une tâche de mémoire à court terme que des non-mots contenant des phonèmes plus rarement associés dans la structure phonologique du français (i.e., / Jybmyl). Cet effet dit de la fréquence phonotactique a une amplitude moindre que l'effet de lexicalité, tout en restant un effet consistant (i.e., Gathercole, Frankish, Pickering, \& Peaker, 1999 ; Majerus, Van der Linden, Mulder, Meulemans, \& Peters, 2004 ; Thorn, Gathercole \& Frankish, 2005). Finalement, au niveau lexico-sémantique, des effets de fréquence lexicale, du degré d'imagerie et du voisinage phonologique ont également été montrés : des mots fréquents, concrets et ayant peu de voisins phonologiques (mots similaires au mot cible et différant par un seul phonème) sont mieux rappelés dans les tâches de mémoire à court terme que des mots peu fréquents, abstraits et ayant beaucoup de voisins phonologiques (Goh \& Pisoni, 2003 ; Hulme et al., 1997 ; Roodenrys et al., 1994 ; Walker \& Hulme, 1999). Tous ces effets témoignent du fait que les connaissances langagières et leur structuration sont intimement liées aux performances dans des tâches de mémoire à court terme verbale. Tous ces effets (à part l'effet du voisinage phonologique qui reste encore peu étudié jusqu'à présent) sont robustes et de même amplitude à travers les différents âges qui ont été étudiés, c'est-à-dire de 4 ans à jusqu'à l'âge adulte (Gathercole et al., 1999 ; Majerus \& Van der Linden, 2003 ; Majerus et al., 2004 ; Pickering \& Gathercole, 2001 ; Roodenrys, Hulme, \& Brown, 1993).

Par ailleurs, les données en neuroimagerie fonctionnelle montrent que les régions sous-tendant le traitement langagier (régions temporales supérieures, moyennes et inférieures) sont activement recrutées lors de la réalisation de tâches de mémoire à court terme et restent actives lors de la phase de maintien alors que les stimuli verbaux à mémoriser ne sont plus physiquement présents (Collette et al., 2001 ; Fiebach, Friederici, Smith, \& Swinney, 2007 ; Majerus et al., 2010 ; Ruchkin et al., 1999). Finalement, les substrats cérébraux de la boucle 
phonologique qui ont été localisés dans la jonction temporo-pariétale gauche, pour le stock phonologique, d'une part, et dans le gyrus frontal inférieur, pour le processus de récapitulation articulatoire, d'autre part, ne sont pas spécifiques à la mémoire à court terme verbale mais sous-tendent eux-mêmes des processus langagiers : la jonction temporo-pariétale est activée pour des tâches de jugement phonologique (jugement de rimes), indépendamment de la charge en mémoire à court terme, et le gyrus frontal inférieur (aire de Broca) est une région fondamentale pour la planification et le contrôle articulatoire de la parole (R. Martin, Wu, Freedman, Jackson, \& Lesch, 2003 ; Paulesu, Firth, \& Frackowiak, 1993 ; Salmon et al., 1996). En conclusion, les tâches de mémoire à court terme verbale sont avant tout des tâches langagières dépendant des mêmes substrats cognitifs et cérébraux que les processus langagiers.

\section{ATTENTION ET MÉMOIRE A COURT TERME VERBALE}

A côté de l'implication des processus langagiers, des travaux récents montrent également l'importance des capacités attentionnelles lors de la réalisation de tâches de mémoire à court terme verbale dites 'passives'. Il est bien admis que les tâches de mémoire à court terme 'actives', telles que les doubles tâches, les tâches de mise à jour ou toute autre tâche nécessitant la manipulation de l'information stockée en mémoire à court terme, font appel à des processus de contrôle attentionnel. Le modèle de la mémoire de travail de Baddeley et Hitch (1974) postule en effet l'existence d'un système de supervision attentionnel qui intervient lors des tâches de mémoire de travail. Ce système attentionnel n'est pas censé jouer de rôle majeur dans les tâches d'empan classiques, tant que l'information doit être simplement mémorisée et restituée, et non manipulée ou intégrée avec d'autres informations. 
Des travaux récents montrent cependant que même des tâches de mémoire à court terme 'passives', impliquant seulement le maintien temporaire de l'information verbale, font déjà intervenir des processus attentionnels. Dans une étude explorant les relations entre mémoire à court terme verbale, attention sélective et développement du vocabulaire auprès d'enfants de 6 à 7 ans, Majerus, Heiligenstein, Gautherot, Poncelet et Van der Linden (2009) ont montré que des tâches d'attention sélective auditive (détecter des mots cibles dans une séquence auditive de mots) expliquaient plus de $30 \%$ de la variance des performances dans une tâche de mémoire à court terme verbale. Par ailleurs, plus de 30\% de la variance dans les scores de vocabulaire des enfants étaient expliqués de façon commune par la tâche attentionnelles et la tâche de mémoire à court terme verbale (voir également Cowan et al., 2005 pour des résultats similaires). Ces résultats suggèrent que l'attention sélective est un médiateur important des performances dans les tâches de mémoire à court terme. Ceci est également soutenu par des données obtenues auprès de l'adulte : Todd, Fougnie et Marois (2005) ainsi que Fougnie et Marois (2007) ont observé que plus le nombre d'éléments à retenir dans une tâche de mémoire à court terme augmente, moins il est probable que le participant détecte un stimulus nouveau présenté brièvement et de façon inattendue dans son champ visuel. Selon les auteurs, ces résultats suggèrent que les tâches de mémoire à court terme absorbent nos capacités attentionnelles de type «task-related attention » : plus la tâche est difficile, plus nos capacités attentionnelles seront monopolisées par cette tâche, et moins elles seront disponibles pour traiter une nouvelle information non reliée à la tâche en cours.

Les données de neuroimagerie fonctionnelle renvoient également à une implication de facteurs généraux, telles que les capacités attentionnelles, dans les tâches de mémoire à court terme verbale. Les régions les plus communément recrutées lors de tâches de mémoire à court terme verbale sont situées dans le lobule pariétal inférieur, et plus précisément le sillon intrapariétal, ainsi que dans le cortex dorso-latéral préfrontal. Ces régions, contrairement aux 
régions temporo-pariétales associées avec la boucle phonologique, sont sensibles à la charge en mémoire à court terme : plus le nombre d'éléments à stocker est élevé, plus l'activité est importante (Ravizza, Delgado, Chein, Becker, \& Fiez, 2004 ; Todd \& Marois, 2004).

L'élément critique ici est que le même réseau fronto-pariétal s'active à la fois lors de tâches de mémoire à court terme verbale et de tâches de mémoire à court terme visuo-spatiale. Une étude récente a comparé le maintien à court terme de séquences de non-mots (pour évaluer la mémoire à court terme verbale) et de séquences de visages non-familiers (pour évaluer la mémoire à court terme visuo-spatiale) : une analyse en conjonction nulle, mettant en évidence les régions activées strictement en commun dans les deux types de tâches de mémoire à court terme, a indiqué une activation bilatérale du sillon intrapariétal et du cortex dorso-latéral préfrontal (Majerus et al., 2010). Ces résultats confirment les résultats obtenus par de nombreuses études ayant exploré de façon isolée les substrats cérébraux associés à la mémoire à court terme verbale ou à la mémoire à court terme visuo-spatiale, et ayant chaque fois identifié le même type de réseau fronto-pariétal, que les stimuli à retenir étaient des mots, des lettres, des visages, des couleurs, ou des figures abstraites (Chen \& Desmond, 2005 ; Linden et al., 2003 ;Majerus et al., 2006, 2007, 2008 ; Salmon et al., 1996 ;Todd et al., 2005). L'ensemble de ces données suggère d'une part que la mémoire à court terme verbale et la mémoire à court terme visuo-spatiale ont beaucoup plus de processus en commun tel que postulé initialement dans le modèle de la mémoire de travail qui opère une distinction nette entre ces deux fonctions de stockage; d'autre part, ces processus en commun sont en partie de nature attentionnelle et renvoient au concept de 'task-related attention' exploré dans les études de Todd et al. (2005) que nous avons décrites.

\section{ORDRE SERIEL ET MÉMOIRE A COURT TERME}


L'aspect qui justifie peut-être le plus l'existence d'un système de mémoire à court terme verbale spécifique est le traitement de l'ordre sériel. Dans une tâche d'empan classique, deux types d'information doivent en effet être maintenus et rappelés : les items présentés

(information «item ») et l'ordre dans lequel les items ont été présentés (information « ordre sériel »). Des études récentes ont montré que la distinction entre information «item » et information « ordre sériel » est capitale pour mieux comprendre la nature des processus sousjacents aux tâches de mémoire à court terme verbale. Des études de Poirier et Saint-Aubin ont en effet montré que les effets des connaissances langagières décrits ci-dessus sont les plus importants pour le rappel de l'information « item » (mesuré par le nombre d'erreurs d'item : omission, paraphasies, intrusions), par rapport au rappel de l'information « ordre sériel » (mesuré par le nombre d'erreurs d'ordre : items de la liste rappelés dans des positions sérielles incorrectes) (Nairne \& Kelley, 2004 ; Poirier \& Saint-Aubin, 1995, 1996 ; Saint-Aubin \& Poirier, 1999). En d'autres termes, les connaissances langagières affectent surtout le rappel des items, mais beaucoup moins le rappel de l'ordre dans lequel les items ont été présentés.

Ainsi, si la mémoire à court terme verbale garde une spécificité en tant que système distinct des connaissances langagières, alors c'est peut-être justement à cause du stockage de l'information « ordre sériel ». Cette hypothèse a été au centre d'une série d'études développementales explorant les liens entre mémoire à court terme verbale et développement du niveau du vocabulaire (voir par exemple, Majerus, 2009, pour une synthèse). La logique sous-jacente à ces études était la suivante : s'il existe un lien causal entre capacités de la mémoire à court terme verbale et l'acquisition du vocabulaire, tel que postulé par un certain nombre d'auteurs (Baddeley, Gathercole \& Papagno, 1998 ; Gathercole, Willis, Emslie, \& Baddeley, 1992) alors un lien devrait être spécifiquement observé pour la composante «ordre sériel » et le niveau de vocabulaire ; un lien entre la composante « item » et le niveau de vocabulaire refléterait simplement la mesure commune du niveau de développement des 
connaissances langagières, étant donné que le rappel de l'information « item » est fortement dépendant des connaissances langagières. Par ailleurs, certains modèles théoriques récents postulent que la capacité à stocker l'ordre de phonèmes en mémoire à court terme détermine la vitesse d'apprentissage à long terme de nouvelles séquences verbales, lesquelles consistent surtout dans un ordonnancement nouveau de phonèmes déjà connus (Gupta, 2003 ; Majerus et al., 2009). En effet, le nombre de phonèmes est fini dans tous les systèmes phonologiques des langues, par contre l'ordre d'agencement de ces mêmes phonèmes permet de distinguer un mot d'un autre mot de même longueur et de même structure syllabique.

Dans plusieurs études, nous avons observé que le rappel de l'information « ordre sériel » est associé de façon systématique au niveau de vocabulaire auprès d'enfants de 6 ans et de 7 ans, alors que est moins le cas pour le rappel de l'information « item » (Majerus, Poncelet, Greffe, \& Van der Linden, 2006 ; Majerus et al., 2009). Par ailleurs, les capacités de rappel de l'information « ordre sériel » mesurées auprès d'enfants âgés de 4 ans prédisent leur niveau de vocabulaire ultérieur (quand ils sont âgés de 5 ans); ceci n'est pas le cas pour les capacités de rappel de l'information « item », une fois le niveau de vocabulaire initial contrôlé (Leclercq \& Majerus, 2010). Ces résultats ont également été reproduits auprès de sujets adultes : les sujets adultes qui présentent les capacités de rappel de l'information « ordre sériel » les plus élevées sont également ceux qui apprennent un nouveau vocabulaire le plus rapidement, après contrôle du niveau intellectuel des participants (Majerus, Poncelet, Elsen, \& Van der Linden, 2006 ; Majerus, Poncelet, Van der Linden, \& Weekes, 2008). En même temps, nous avons observé que le rappel de l'information «item » est influencé par les connaissances langagières existantes (Majerus, Poncelet, et al., 2008). De la même façon, les donnés en neuroimagerie fonctionnelle ont révélé que le maintien à court terme de l'information « item » active les régions temporales supérieures, moyennes et inférieures impliquées dans le traitement langagier alors que le maintien de l'information « ordre sériel » 
active un circuit spécifique incluant la partie antérieure du sillon intrapariétal droit (Majerus et al., 2006, 2010). Ces mêmes études ont également montré que le sillon intrapariétal gauche est activé à la fois pour le maintien des informations «item » et «ordre sériel », confirmant la fonction plus générale exercée par cette région, comme nous l'avons discuté dans la section précédente.

Sur base de ces travaux, nous pouvons retenir que les tâches de mémoire à court terme verbale mesurent à la fois le maintien de l'information « item » ainsi que le maintien de l'information « ordre sériel », le premier étant déterminé en grande partie par les connaissances langagières existantes, et le deuxième étant sous-tendu par un système plus spécifique, indépendant des connaissances langagières mais contribuant à l'apprentissage de nouvelles séquences verbales. Ainsi, la capacité à stocker l'information « ordre sériel » est un troisième facteur à prendre en compte lors de la compréhension du développement des capacités de la mémoire à court terme verbale chez l'enfant. Même si les études à ce sujet sont encore rares, quelques travaux ont révélé que la précision d'encodage de l'information « ordre sériel » augmente avec l'âge, l'amplitude des erreurs d'ordre (le nombre de positions qu'un item migre lors du rappel par rapport à sa position de départ) diminuant avec l'âge (McCormack, Brown, Vousden, \& Henson, 2000).

\section{IMPLICATIONS THEORIQUES}

Face à l'ensemble de ces données, le modèle initial de la boucle phonologique a progressivement fait place à de nouveaux modèles intégrant, en tout ou en partie, les facteurs identifiés dans cette revue. D'abord, une série de modèles de la mémoire à court terme verbale ont été développés en prenant comme point de départ le système langagier (Burgess \& Hitch, 2006 ; Gupta, 2003 ; N. Martin \& Saffran, 1992) ou en connectant la boucle phonologique au 
système langagier (Baddeley et al., 1998). Ces modèles peuvent rendre compte de l'influence des connaissances phonologiques, lexicales et sémantiques sur les performances de rappel dans les tâches de mémoire à court terme verbale, certains modèles adoptant des positions même très radicales à ce sujet : N. Martin et Saffran (1992) considèrent que la mémoire à court terme verbale n'existe pas en tant que telle mais qu'elle résulte simplement de l'activation temporaire et de la dégradation rapide de cette activation du système langagier, où les représentations phonologiques, lexicales et sémantiques correspondant aux mots à mémoriser s'activent et se désactivent. Ces activations s'effectuent de façon plus rapide et plus robuste pour des mots familiers, fréquents et concrets ayant les représentations lexicales et sémantiques les plus riches et les plus solides.

Ces formes de modèles linguistiques de la mémoire à court verbale ne tiennent cependant pas compte du traitement de l'ordre sériel. Des modèles plus nuancés ont ainsi été développés, considérant que l'information «item » est traitée et stockée via l'activation du système du langagier, alors que le traitement et le maintien de l'information « ordre sériel » s'effectue via un système spécifique, encodant l'ordre sériel sur base de codes temporels, contextuels, positionnels ou énergétiques ; ce système se trouve en interaction étroite avec le système langagier et d'une part, encode l'ordre dans lequel les items ont été activés dans le système langagier, et d'autre part réactive les items selon leur ordre de présentation initial lors de la phase de rappel (e.g., Brown, Preece, \& Hulme, 2000 ; Burgess \& Hitch, 2006 ; Gupta, 2003). Alors que ces modèles intègrent la distinction entre information « item » et « ordre sériel », tout en réservant une place importante au système langagier, aucun de ces modèles ne tient compte des facteurs attentionnels que nous avons identifiés.

Les modélisations de la mémoire à court terme qui tiennent compte de ces facteurs attentionnels sont très rares, et seul le cadre théorique du focus attentionnel de Cowan (1995 ; 1999) réserve une place explicite aux capacités attentionnelles. Rappelons que le modèle de 
Baddeley (1986) considère également l'existence de capacités attentionnelles, mais celles-ci sont censées intervenir surtout lors de tâches de mémoire de travail, et non lors du simple stockage passif. Ainsi, Cowan considère, tout comme une partie des modèles cités ci-dessus, que la mémoire à court terme verbale résulte de l'activation temporaire des connaissances à long terme stockées dans le système langagier. Cependant, contrairement aux modèles décrits ci-dessus, Cowan estime que des capacités attentionnelles sont nécessaires pour le maintien de ces activations temporaires dans ce qu'il qualifie de 'foyer attentionnel'. Pour Cowan, nous pouvons maintenir dans notre foyer attentionnel qu'un nombre très limité d'éléments (4 éléments), ce nombre pouvant être augmenté via des processus de chunking. Ce modèle tient compte de l'influence des capacités de focalisation attentionnelle dans les tâches de mémoire à court terme verbale, ainsi que de l'influence des connaissances langagières. Cependant, aucun mécanisme explicite n'est prévu pour le traitement et le stockage de l'ordre sériel. D’autres modèles attentionnels ont été développés plus spécifiquement pour les tâches de mémoire de travail, mais certains de leurs postulats sont similaires à celles de Cowan pour les tâches de mémoire à court terme. Par exemple, pour Barrouillet, Bernardin et Camos (2004) le maintien des informations, que ce soit dans des tâches de mémoire à court terme ou des tâches de mémoire de travail, dépend de capacités attentionnelles contrôlées ; sans ce contrôle attentionnel des informations à mémoriser, les informations se dégradent très rapidement (voir également Engle, Kane et Tuholski (1999). Cependant, tout comme Cowan, le problème du stockage de l'ordre sériel n'est pas considéré de façon explicite par ces modèles attentionnels de la mémoire de travail.

Les données présentées ici indiquent donc que de nouveaux modèles théoriques de la mémoire à court terme doivent être développés intégrant à la fois les interactions avec le système langagier, les interactions avec les capacités attentionnelles et le traitement de l'ordre sériel. Dans cette optique, Majerus et al. (2009 ; Majerus, 2009, 2010) ont proposé un modèle 
à trois composantes de la mémoire à court terme, le modèle A-O-STM (voir Figure 1). Dans ce modèle, l'attention sélective prend une place centrale et interagit avec le système langagier pour l'encodage et le maintien de l'information « item »d'une part, et avec un système assurant le traitement de l'ordre sériel d'autre part. Par ailleurs, un lien bidirectionnel entre systèmes langagier et du traitement de l'ordre sériel permet de coupler chaque item activé dans le système langagier avec sa position sérielle encodé par le système de traitement de l'ordre sériel. Ce lien bidirectionnel permet également de rendre compte de l'importance des capacités de traitement de l'ordre sériel pour l'apprentissage de nouvelles séquences verbales activées dans le système langagier, en permettant, lors de la présentation d'un nouveau mot, de réactiver la nouvelle séquence de phonèmes dans le système phonologique, conduisant progressivement vers l'établissement d'une représentation phonologique plus stable, par exemple via des processus d'apprentissage de type apprentissage de Hebb à l'intérieur du système langagier (e.g ., Burgess \& Hitch, 2006 ; Gupta, 2003). La nature du code utilisé pour représenter l'information « ordre sériel » reste cependant encore non-spécifié et devra faire l'objet de travaux de recherche futurs.

\section{$<$ INSERER FIGURE 1 ICI >}

En ce qui concerne plus spécifiquement le rôle de la variable «attention sélective », les informations « item » et « ordre sériel » sont représentées et activées temporairement dans leurs systèmes correspondants, et les capacités d'attention sélective permettent de maintenir cette activation dans le temps. Ces capacités attentionnelles étant limitées, la quantité d'information qui pourra être maintenue à un moment donné sera limitée, tout comme dans Cowan (1999). Par ailleurs, l'attention peut être focalisée sur les systèmes langagiers et de traitement de l'ordre sériel de façon équivalente, ou davantage sur l'un ou l'autre des deux systèmes, en fonction des exigences de la tâche de mémoire à court terme : une tâche ne nécessitant que le maintien et le rappel de l'information «item » (par exemple, rappeler tous 
les mots présentés, indépendamment de leur position sérielle) permettrait de dédier les capacités attentionnelles exclusivement aux activations ayant eu lieu dans le système langagier, favorisant leur maintien au détriment des informations encodées dans le système de traitement de l'ordre sériel.

\section{IMPLICATIONS POUR L'EVALUATION DE LA MÉMOIRE A COURT TERME}

\section{VERBALE}

Face aux différents facteurs identifiés dans cette revue, il apparaît clairement qu'une tâche de mémoire à court terme verbale, aussi simple soit-elle, ne mesure pas simplement les capacités d'un système spécialisé pour le stockage à court terme telle que la boucle phonologique.

L'intrication des facteurs attentionnels, langagiers et sériels rend donc difficile l'interprétation de performances faibles présentées par un enfant ou un adulte aux tâches d'empan. Il est cependant possible, avec des procédés relativement simples, de tenter de dissocier l'influence de ces trois facteurs.

Une première approche, qui n'exige pas d'apporter de modifications aux tâches d'empan déjà existantes, consiste à réaliser une analyse plus fine des erreurs commises. Nous avons vu que les connaissances langagières affectent surtout le rappel de l'information « item », alors que le rappel de l'information « ordre sériel » peut être considéré comme reflétant de ce qui est le plus spécifique à un système de mémoire à court terme verbal. Dans cette optique, la comptabilisation des erreurs commises par un enfant lors d'une tâche d'empan peut s'avérer très utile. Une augmentation importante des erreurs « item » (omissions, paraphasies sémantiques et/ou phonologiques) indiquera plutôt un problème au niveau de l'interaction avec les connaissances langagières alors qu'une augmentation importante des erreurs de position sérielle (item correct rappelé mais pas dans le bon ordre par 
rapport à la série de départ) indiquera davantage un problème au niveau du traitement et du stockage de l'ordre sériel. Conformément à cette proposition, des patients ayant une dégradation des connaissances langagières présentent une augmentation importante des erreurs «item », alors que le nombre d'erreurs d'ordre reste inchangé, voire diminue dans les tâches de rappel sériel immédiat (tâches identiques aux tâches d'empan sauf que le même nombre listes est administré à tous les participants et qu'il n'y pas de règle d'arrêt comme pour l'empan ; Majerus, Norris, \& Patterson, 2007). Il faut cependant noter qu'une tâche d'empan classique utilisant des séries de chiffres n'est pas idéale pour évaluer le rappel de l'information « item » dans la mesure où les chiffres utilisés (1 à 9) constituent un ensemble d'items finis, faisant en sorte que pour les longueurs de listes plus élevées ( 7 à 9 chiffres), la probabilité de se tromper au niveau de l'information « item » est relativement faible. La même remarque vaut pour le rappel de listes de mots où les mots sont sélectionnés de façon répétitive à partir d'un ensemble limité de 8 ou 9 mots : au fur et à mesure des essais, le participant sait quels mots vont être présentés et il doit surtout retenir leur ordre d'apparition. Ainsi, le test maximal pour le rappel de l'information « item » consiste en la présentation de listes de mots où chaque mot est utilisé qu'une seule fois sur l'ensemble de la tâche. Par ailleurs, ces listes peuvent explicitement faire varier l'influence possible des connaissances phonologiques, lexicales et sémantiques, en comparant par exemple le rappel pour des listes de mots versus de non-mots, ou pour des listes de mots fréquents versus peu fréquents, ou encore pour des listes de mots abstraits versus concrets. Des études ont montré que des patients ayant des difficultés spécifiques au niveau de la représentation de l'information phonologique vont présenter des effets de lexicalité exagérés : le rappel de listes de mots peut être dans les normes mais le rappel de listes de non-mots sera sévèrement altéré (e.g., Freedman \& R. Martin, 2001 ; Hanten \& R. Martin, 2000 ; Majerus, Van der Linden, Poncelet, \& Metz-Lutz, 2004). D’un autre côté, des patients ayant des difficultés spécifiques 
au niveau du traitement lexical et sémantique vont présenter des effets de lexicalité amoindris : le rappel de listes de mots sera au même niveau que le rappel de listes de nonmots (e.g., Freedman \& R. Martin, 2001 ; Majerus, Norris, \& Patterson, 2007).

Le rappel de l'information « item » peut également être évalué par des tâches plus spécifiques, délibérément construites afin de maximiser la rétention à court terme de l'information « item ». Ces tâches soit ne présentent qu'un seul item à répéter après un délai durant lequel l'enfant doit répéter 'blablablabla...' afin d'éviter la récapitulation articulatoire, soit impliquent que seule l'information « item » doive être reconnue, telles que les tâches d'empan de rimes ou d'empan de catégories (Majerus, Poncelet, Greffe et al., 2006 ; Hanten \& R. Martin, 2000). Dans ces dernières tâches, des listes de mots de longueur croissante sont présentées, suivies d'un nouvel mot, et l'enfant doit décider si ce mot rime avec un des mots de la liste (empan de rimes) ou fait partie de la même catégorie sémantique qu'un des mots de la liste (empan de catégories). Ces deux tâches permettent d'évaluer la mémoire à court terme pour l'information « item » spécifiquement au niveau phonologique, d'une part, et au niveau sémantique, d'autre part. Ces tâches nécessitent cependant que l'enfant maîtrise les concepts de rime et de catégorie sémantique, et elles ne sont donc guère applicables avant l'âge de 8 ans (e.g., Majerus et al., 2005).

Le rappel de l'information « ordre sériel », à part d'être estimé à partir des erreurs de position sérielle dans certaines des tâches décrites ci-dessus, peut également être évalué par des tâches spécifiquement construites afin de maximiser le rappel de cette information. L'adaptation la plus simple consiste en une tâche d'empan de chiffres classique légèrement modifiée. Le principe dans cette adaptation est que l'information «item » soit connue à l'avance ; ceci peut être obtenu en informant l'enfant au préalable des chiffres qui seront présentés : les séries de 3 chiffres comporteront toujours les chiffres de 1 à 3 , les séries de 4 chiffres comporteront toujours les chiffres de 1 à 4, etc.... Par ailleurs, au moment du rappel, 
on donne à l'enfant des cartons représentant les chiffres présentés et uniquement ceux-là : l'enfant doit simplement ordonner les cartes selon l'ordre d'apparition des chiffres dans la liste. Cette procédure maximise la rétention et le rappel de l'information « ordre sériel » tout en minimisant celui de l'information « item ». Ces tâches sont applicables chez l'enfant dès que la chaîne numérique de base est acquise et automatisée. Pour des enfants plus jeunes (par exemple, 4 ans), une variante utilisant des ensembles de noms d'animaux d'un âge d'acquisition précoce (tels que chien, chat, coq, lion, etc...) a été développée (Majerus, Poncelet, Greffe et al., 2006 ; Leclercq \& Majerus, 2010). Cette tâche, présentée sous forme de jeu («la course des animaux »), consiste à dire à l'enfant qu'il va entendre le nom de plusieurs animaux faisant une course et qu'il doit reproduire, avec des cartes représentant ces animaux, l'ordre dans lequel les animaux sont arrivés à la ligne d'arrivée : la carte du vainqueur (premier animal) sera mis sur la marche la plus élevée du podium des vainqueurs, la carte du deuxième animal sera mis sur la marche suivante, etc... En utilisant ce type de tâches, Majerus, Van der Linden, Braissand et Eliez (2007) ont pu montrer que des enfants souffrant d'une microdélétion du chromosome 22q11.2 (syndrome vélo-cardio-facial) présentent un déficit spécifique pour le rappel de l'ordre sériel dans les tâches de mémoire à court terme verbale.

Finalement, l'influence des facteurs attentionnels peut être estimée d'au moins deux façons. Premièrement, en cas de performances faibles dans les tâches de mémoire à court terme verbale, touchant de façon équivalente le rappel de l'information «item » et celui de l'information « ordre sériel », une diminution des facteurs attentionnels peut être suspectée. Les capacités d'attention sélective peuvent également être évaluées de façon plus directe par des tâches d'attention sélective pour des informations auditives verbales : ces tâches consistent à repérer des mots cibles dans des séquences continues de mots d'une durée de 90 secondes par exemple. La plupart des batteries des fonctions attentionnelles mesurant 
davantage l'attention visuelle que l'attention auditivo-verbale, Majerus et al. (2009) ont développé une tâche de ce type qui peut être administré auprès d'enfants à partir de l'âge de 6 ans et qui explique jusqu'à 30\% de la variance dans les tâches de mémoire à court terme verbale.

\section{CONCLUSIONS}

Le domaine de recherche autour de la mémoire à court terme verbale est un domaine actuellement particulièrement dynamique. Malgré l'impact toujours important du modèle original de la boucle phonologique de Baddeley et Hitch (1974), de multiples conceptions théoriques alternatives ont vu le jour. Quasi aucun modèle de la mémoire à court terme récent n'ignore plus le rôle important joué par les systèmes langagiers, lesquels constituent en réalité la base de toute mémorisation à court terme d'informations verbales. Nous ne saurions mémoriser à court terme ce que nous ne savons pas traiter et représenter dans nos systèmes phonologiques et lexico-sémantiques... Par contre, les théories attentionnelles et celles s'intéressant plus spécifiquement au stockage de l'information « ordre sériel » évoluent actuellement sur des voies de recherche parallèles sans se croiser directement. L'objectif de cette revue était de démontrer l'importance de la triade « système langagier - ordre sériel attention sélective » comme principe de base de la mémorisation à court terme. Un modèle théorique intégrant ces trois éléments et leurs interactions possibles a été présenté. Les implications pour le développement de la mémoire à court terme et des interactions entre mémoire à court terme et développement langagier ont également été appréhendées.

Cependant, le modèle intégratif présenté ici fait actuellement encore l'impasse sur un autre processus qui intervient également lors de la mémorisation à court terme telle que la sensibilité à l'interférence, l'interférence pouvant provenir des items à l'intérieur de chaque 
essai, des items d'un essai à l'autre, ou encore de l'interférence entre l'output (les items produits lors du rappel) et ceux maintenus en mémoire à court terme (Hamilton \& Martin, 2007 ; Oberauer, 2003). La résistance à l'interférence pourrait faire partie intégrante des systèmes de traitement langagier, comme proposé par certains auteurs (Barde, Schwartz, Chrysikou, \& Thompson-Schill, 2010 ; Hamilton \&Martin, 2007), ou être sous-tendu par des processus exécutifs spécifiques sous-tendus par le cortex préfrontal dorso-latéral (Postle, 2005). Des travaux futurs vont permettre de préciser davantage ces questions.

Il est néanmoins évident déjà actuellement qu'une simple tâche de mémoire à court terme, tel que l'empan de chiffres ou l'empan de mots, ne met pas en jeu un système de stockage bien spécifique, mais est déjà le résultat de l'interaction de multiples systèmes, incluant des systèmes de connaissances à long terme (langage) et des systèmes davantage exécutifs (contrôle attentionnel, interférence), rendant la distinction entre tâches d'empan simples (empan de chiffres) et tâches d'empan complexes (tâches de mise à jour, doubles tâches) assez artificielle. 


\section{RÉFÉRENCES}

Baddeley, A. D. \& Hitch, G. J. (1974). Working memory. In G.H.Bower (Ed.), The psychology of learning and motivation (pp. 47-90). San Diego, CA: Academic Press.

Baddeley, A. (1986). Working memory. Oxford, England UK: Clarendon Press/Oxford University Press.

Baddeley, A., Gathercole, S., \& Papagno, C. (1998). The phonological loop as a language learning device. Psychological Review, 105, 158-173.

Barde, L. H. F., Schwartz, M. F., Chrysikou, E. G., \& Thompson-Schill, S. L. (2010). Reduced short-term memory span in aphasia and susceptibility to interference: Contribution of material-specific maintenance deficits. Neuropsychologia, 48, 909920.

Barrouillet, P., Bernardin, S., \& Camos, V. (2004). Time constraints and resource sharing in adults' working memory spans. Journal of Experimental Psychology: General, 133, 83-100.

Brown, G. D. A., Preece, T., \& Hulme, C. (2000). Oscillator-based memory for serial order. Psychological Review, 107, 127-181.

Burgess, N. \& Hitch, G. J. (2006). A revised model of short-term memory and long-term learning of verbal sequences. Journal of Memory and Language, 55, 627-652.

Chen, S. H. \& Desmond, J. E. (2005). Temporal dynamics of cerebro-cerebellar network recruitment during a cognitive task. Neuropsychologia, 43, 1227-1237. 
Collette, F., Majerus, S., Van der Linden, M., Dabe, P., Degueldre, C., Delfiore, G. et al. (2001). Contribution of long-term memory to verbal short-term memory tasks: A PET activation study. Memory, 9, 249-259.

Cowan, N. (1995). Attention and memory: An integrated framework. New York: Oxford University Press.

Cowan, N., Wood, N. L., Wood, P. K., Keller, T. A., Nugent, L. D., \& Keller, C. V. (1998). Two separate verbal processing rates contributing to short-term memory span. Journal of Experimental Psychology: General, 127, 141-160.

Cowan, N. (1999). An embedded-processes model of working memory. In A.Miyake \& P. Shah (Eds.), Models of working memory: Mechanisms of active maintenance and executive control (pp. 62-101). Cambridge: Cambridge University Press.

Cowan, N., Elliott, E. M., Saults, J. S., Morey, C. C., Mattox, S., Hismjatullina, A. et al. (2005). On the capacity of attention: Its estimation and its role in working memory and cognitive aptitudes. Cognitive Psychology, 51, 42-100.

Engle, R. W., Kane, M. J., \& Tuholski, S. W. (1999). Individual differences in working memory capacity and what they tell us about controlled attention, general fluid intelligence, and functions of the prefrontal cortex. In A.Miyake \& P. Shah (Eds.), Models of working memory: Mechanisms of active maintenance and executive control (pp. 102-134). Cambridge: Cambridge University Press.

Fiebach, C. J., Friederici, A. D., Smith, E. E., \& Swinney, D. (2007). Lateral Inferotemporal Cortex Maintains Conceptual-Semantic Representations in Verbal Working Memory. Journal of Cognitive Neuroscience, 19, 2035-2049. 
Fougnie, D. \& Marois, R. (2007). Executive working memory load induces inattentional blindness. Psychonomic Bulletin and Review, 14, 142-147.

Freedman, M. L. \& Martin, R. C. (2001). Dissociable components of short-term memory and their relation to long-term learning. Cognitive Neuropsychology, 18, 193-226.

Gathercole, S. E. (2002). Memory development during childhood years. In A.D.Baddeley, M. D. Kopelman, \& B. A. Wilson (Eds.), The Handbook of Memory Disorders (pp. 475500). Chichester, UK: John Wiley \& Sons Ltd.

Gathercole, S. E., Adams, A. M., \& Hitch, G. J. (1994). Do young children rehearse? An individual-differences analysis. Memory and Cognition, 22, 201-207.

Gathercole, S. E., Frankish, C. R., Pickering, S. J., \& Peaker, S. (1999). Phonotactic influences on short-term memory. Journal of Experimental Psychology: Human Learning and Memory, 25, 84-95.

Goh, W. D. \& Pisoni, D. B. (2003). Effects of lexical competition on immediate memory span for spoken words. Quarterly Journal of Experimental Psychology, 56A, 929-954.

Gupta, P. (2003). Examining the relationship between word learning, nonword repetition and immediate serial recall in adults. Quarterly Journal of Experimental Psychology, 56A, 1213-1236.

Hamilton, A. \& Martin, R. C. (2007). Proactive interference in a semantic short-term memory deficit: Role of semantic and phonological relatedness. Cortex, 43, 112-123.

Hanten, G. \& Martin, R. C. (2000). Contributions of phonological and semantic short-term memory to sentence processing: Evidence from two cases of closed-head injury in children. Journal of Memory and Language, 43, 335-361. 
Hitch, G. \& Halliday, M. S. (1983). Working memory in children. Philosophical Transactions of the Royal Society of London B, 302, 324-340.

Hulme, C., Maughan, S., \& Brown, G. D. (1991). Memory for familiar and unfamiliar words: Evidence for a long-term memory contribution to short-term memory span. Journal of Memory and Language, 30, 685-701.

Hulme, C., Roodenrys, S., Schweickert, R., Brown, G. D., Martin, S., \& Stuart, G. (1997). Word-frequency effects on short-term memory tasks: evidence for a redintegration process in immediate serial recall. Journal of Experimental Psychology: Learning, Memory, and Cognition, 23, 1217-1232.

Leclercq, A. L. \& Majerus, S. (2010). Serial Order Short-Term Memory Predicts Vocabulary Development: Evidence from a Longitudinal Study. Developmental Psychology, 46, 417-427.

Linden, D. E. J., Bittner, R. A., Muckli, L., Waltz, J. A., Kriegeskorte, N., Goebel, R. et al. (2003). Cortical capacity for visual working memory: dissociation of fMRI load effects in a fronto-parietal network. Neurolmage, 20, 1518-1530.

Majerus, S. \& Van der Linden, M. (2003). The development of long-term memory effects on verbal short-term memory : A replication study. British Journal of Developmental Psychology, 21, 303-310.

Majerus, S., Van der Linden, M., Poncelet, M., \& Metz-Lutz, M. N. (2004). Can phonological and semantic short-term memory be dissociated? Further evidence Landau-Kleffner Syndrome. Cognitive Neuropsychology, 21, 491-512. 
Majerus, S., Van der Linden, M., Mulder, L., Meulemans, T., \& Peters, F. (2004). Verbal short-term memory reflects the sublexical organization of the phonological language network: Evidence from an incidental phonotactic learning paradigm. Journal of Memory and Language, 51, 297-306.

Majerus, S., Amand, P., Bonivers, V., Demanez, L., Demanez, J. P., \& Van der Linden, M. (2005). A quantitative and qualitative assessment of verbal short-term memory and phonological processing in 8-year-olds with a history of repetitive otitis media. Journal of Communication Disorders, 38, 473-498.

Majerus, S., Poncelet, M., Greffe, C., \& Van der Linden, M. (2006). Relations between vocabulary development and verbal short-term memory: the importance of short-term memory for serial order information. Journal of Experimental Child Psychology, 93, 95-119.

Majerus, S., Poncelet, M., Elsen, B., \& Van der Linden, M. (2006). Exploring the relationship between new word learning and short-term memory for serial order recall, item recall and item recognition. European Journal of Cognitive Psychology, 18, 848-873.

Majerus, S., Poncelet, M., Van der Linden, M., Albouy, G., Salmon, E., Sterpenich, V. et al. (2006). The left intraparietal sulcus and verbal short-term memory: Focus of attention or serial order? NeuroImage, 32, 880-891.

Majerus, S., Van der Linden, M., Braissand, V., \& Eliez, S. (2007). Verbal short-term memory in children and adults with a chromosome $22 \mathrm{q} 11.2$ deletion. A specific deficit in serial order retention capacities? American Journal on Mental Retardation, 112, 7993. 
Majerus, S., Norris, D., \& Patterson, K. (2007). What do patients with semantic dementia remember in verbal short-term memory? Sounds and order but not words. Cognitive Neuropsychology, 24, 131-151.

Majerus, S., Bastin, C., Poncelet, M., Van der Linden, M., Salmon, E., Collette, F. et al. (2007). Short-term memory and the left intraparietal sulcus: Focus of attention? Further evidence from a face short-term memory paradigm. NeuroImage, 35, 353-367.

Majerus, S., Poncelet, M., Van der Linden, M., \& Weekes, B. (2008). Lexical learning in bilingual adults:the relative importance of short-term memory for serial order and phonological knowledge. Cognition, in press.

Majerus, S., Belayachi, S., De Smedt, B., Leclercq, A. L., Martinez, T., Schmidt, C. et al. (2008). Neural networks for short-term memory for order differentiate high and low proficiency bilinguals. NeuroImage, 42, 1698-1713.

Majerus, S. (2009). Verbal short-term memory and temporary activation of language representations: the importance of distinguishing item and order information. In A.S.Thorn \& M. Page (Eds.), Interactions between short-term and long-term memory in the verbal domain (pp. 244-276). Hove, UK: Psychology Press.

Majerus, S., Heiligenstein, L., Gautherot, N., Poncelet, M., \& Van der Linden, M. (2009). The impact of auditory selective attention on verbal short-term memory and vocabulary development. Journal of Experimental Child Psychology, 103, 66-86.

Majerus, S., D'Argembeau, A., Martinez, T., Belayachi, S., Van der Linden, M., Collette, F. et al. (2010). The commonality of neural networks for verbal and visual short-term memory. Journal of Cognitive Neuroscience, in press. 
Majerus, S. (2010). Das verbale Kurzzeitgedächtnis als Produkt der Interaktion zwischen Aufmerksamkeitskapazitäten, Sequenzverarbeitung und Aktivierung des Sprachsystems. Psychologische Rundschau, 61, 10-17.

Martin, N. \& Saffran, E. M. (1992). A computational account of deep dysphasia: Evidence from a single case study. Brain and Language, 43, 240-274.

Martin, R. C., Wu, D., Freedman, M., Jackson, E. F., \& Lesch, M. (2003). An event-related fMRI investigation of phonological versus semantic short-term memory. Journal of Neurolinguistics, 16, 341-360.

Martin, R. C., Shelton, J. R., \& Yaffee, L. S. (1994). Language processing and working memory: Neuropsychological evidence for separate phonological and semantic capacities. Journal of Memory and Language, 33, 83-111.

McCormack, T., Brown, G. D., Vousden, J. I., \& Henson, R. N. A. (2000). Children's serial recall errors: implications for theories of short-term memory development. Journal of Experimental Child Psychology, 76, 222-252.

Nairne, J. S. \& Kelley, M. R. (2004). Separating item and order information through process dissociation. Journal of Memory and Language, 50, 113-133.

Oberauer, K. (2003). Understanding serial position curves in short-term recognition and recall. Journal of Memory and Language, 49, 469-483.

Palmer, S. (2000). Working memory: a developmental study of phonological recoding. Memory, 8, 179-193.

Paulesu, E., Frith, C. D., \& Frackowiak, R. S. J. (1993). The neural correlates of the verbal component of working memory. Nature, 362, 342-345. 
Pickering, S. J. \& Gathercole, S. E. (2001). The Working Memory Test Battery for Children. UK: Psychological Corporation.

Poirier, M. \& Saint-Aubin, J. (1995). Memory for related and unrelated words: Further evidence on the influence of semantic factors in immediate serial recall. The Quarterly Journal of Experimental Psychology, 48A, 384-404.

Poirier, M. \& Saint-Aubin, J. (1996). Immediate serial recall, word frequency, item identity and item position. Canadian Journal of Experimental Psychology, 50, 408-412.

Postle, B. R. (2005). Delay-period activity in the prefrontal cortex: One function is sensory gating. Journal of Cognitive Neuroscience, 17, 1679-1690.

Ravizza, S. M., Delgado, M. R., Chein, J. M., Becker, J. T., \& Fiez, J. A. (2004). Functional dissociations within the inferior parietal cortex in verbal working memory. NeuroImage, 22, 562-573.

Roodenrys, S., Hulme, C., \& Brown, G. (1993). The development of short-term memory span: Separable effects of speech rate and long-term memory. Journal of Experimental Child Psychology, 56, 431-442.

Roodenrys, S., Hulme, C., Alban, J., \& Ellis, A. W. (1994). Effects of word frequency and age of acquisition on short-term memory span. Memory and Cognition, 22, 695-701.

Ruchkin, D. S., Berndt, R. S., Johnson, R. Jr., Grafman, J., Ritter, W., \& Canoune, H. L. (1999). Lexical contributions to retention of verbal information in working memory: Event-related brain potential evidence. Journal of Memory and Language, 41, 345364. 
Saint-Aubin, J. \& Poirier, M. (1999). Semantic similarity and immediate serial recall: Is there a detrimental effect on order information? The Quarterly Journal of Experimental Psychology, 52A, 367-394.

Salmon, E., Van der Linden, M., Collette, F., Delfiore, G., Maquet, P., Degueldre, C. et al. (1996). Regional brain activity during working memory tasks. Brain, 119, 1617-1625.

Thorn, A. S., Gathercole, S. E., \& Frankish, C. R. (2005). Redintegration and the benefits of long-term knowledge in verbal short-term memory: An evaluation of Schweickerts (1993) multinomial processing tree model. Cognitive Psychology, 50, 133-158.

Todd, J. J. \& Marois, R. (2004). Capacity limit of visual short-term memory in human posterior parietal cortex. Nature, 428, 751-754.

Todd, J. J., Fougnie, D., \& Marois, R. (2005). Visual short-term memory load suppresses temporo-parietal junction activity and induces inattentional blindness. Psychological Science, 16, 965-972.

Walker, I. \& Hulme, C. (1999). Concrete words are easier to recall than abstract words: Evidence for a semantic contribution to short-term serial recall. Journal of Experimental Psychology: Learning, Memory, and Cognition, 25, 1256-1271. 
Figure 1. Le modèle A-O-STM, un modèle de la mémoire à court terme verbale se basant sur l'activation temporaire du système langagier, le traitement de l'ordre sériel et l'attention sélective.

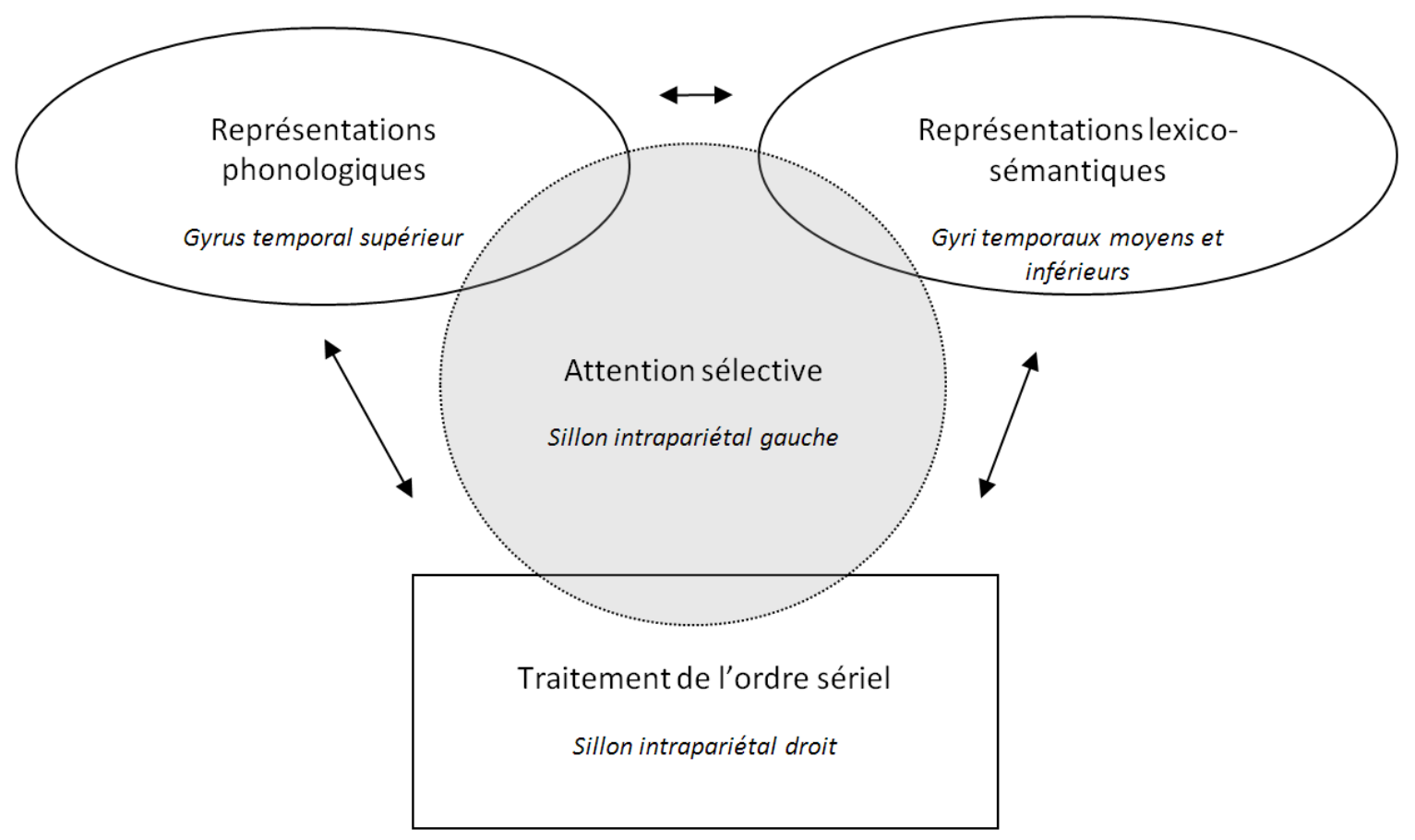

